\title{
DAS TIERREICH
}

Eine Zusammenstellung und Kennzeichnung der rezenten Tierformen

HeINZ WeRmuth

(Wirbeltiere)

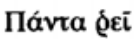

Herausgeber

\section{EDwin MöHN}

(Wirbellose)

Sine systemate chaos

\section{Lieferung 101}

Liste der rezenten Amphibien und Reptilien

\section{Anomalepididae, Leptotyphlopidae, Typhlopidae}

Bearbeitet von

Donald E. Hahn (Cottonwood, Arizona)

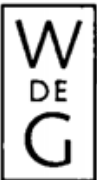

Walter de Gruyter · Berlin · New York

1980 
ISBN 3110068606

(9) 1980 by Walter de Gruyter \& Co., vormals G. J. Göschen'sche Verlagshandlung. J. Guttentag, Verlagsbuchhandlung Georg Reimer, Karl J. Trübner, Veit \& Comp., Berlin 30.

Alle Rechte, insbesondere das Recht der Vervielfaltigung und Verbreitung, sowie der Ubersetzung, vorbehalten. Kein Teil des Werkes darf in irgendeiner Form (durch Photokopie, Mikrofilm oder ein anderes Verfahren) ohne schriftliche Genehmigung des Verlages reproduriert oder unter Verwendung elektronischer Systeme verarbeitet, vervielfältigt oder verbreitet werden.

$$
\text { Printed in Germany. }
$$

Satz: IBM-Composer Walter de Gruyter \& Co., Berlin.

Druck: Karl Gerike, Berlin.

Bindearbeiten: Dieter Mikolai, Berlin. 
Liste der rezenten Amphibien und Reptilien

\title{
Anomalepididae, Leptotyphlopidae, Typhlopidae
}

\author{
Bearbeitet von
}

Donald E. Hahn 

Die Liste der rezenten Amphibien und Reptilien ist Herrn Prof. Dr. Robert Mertens $\dagger$, Direktor emeritus des Natur-Museums und Forschungs-Institutes Senckenberg zu Frankfurt am Main, als Dank der Fachkollegen für seine überragenden Verdienste um die Herpetologie gewidmet. 
Als Beiträge zur Liste der rezenten Amphibien und Reptilien sind bisher erschie. nen:

Amphibia: G y m n o ph i o n a (GORHAM, Ottawa: Lieferung 78,1962$)$. - S a li e n t i a Ascaphidae, Leiopelmatidae, Pipidae, Discoglossidae, Pelobatidae, Leptodactylidae, Rhinophrynidae (GORHAM, St. John: Lieferung 85, 1966); Hylidae (DUELLMAN, Lawrence: Lieferung 95, 1977).

Reptilia: Testudines, Crocodylia, Rhy n chocephalia (WERMUTH, Ludwigsburg, \& MERTENS, Frankfurt am Main: Lieferung 100, 1977). - S a u r i a : Gekkonidae, Pygopodidae, Xantusiidae (WERMUTH, Ludwigsburg: Lieferung 80, 1965); Agamidae (WERMUTH, Ludwigsburg: Lieferung 86, 1967); Chamaeleonidae (MERTENS, Frankfurt am Main: Lieferung 83, 1966); Cordylidae - Cordylinae, Chamaesaurinae - (WERMUTH, Ludwigsburg: Lieferung 87, 1968); Anguidae, Anniellidae, Xenosauridae (WERMUTH, Ludwigsburg: Lieferung 90, 1969); Helodermatidae, Varanidae, Lanthanotidae (MERTENS, Frankfurt am Main: Lieferung 79,1963). - S e r p e n t e s: Anomalepididae, Leptotyphlopidae, Typhlopidae (HAHN, Cottonwood: Lieferung 101, 1980); Boidae - Boinae, Bolyeriinae, Loxoceminae, Pythoninae - (STIMSON, London: Lieferung 89, 1969); Uropeltidae (GANS, Buffalo: Lieferung 84, 1966); Colubridae - Dipsadinae - (PETERS, Washington: Lieferung 81, 1965).

Als weitere Beiträge erscheinen demnächst:

Amphibia: C a u d a t a (BRAME, Pasadena, \& Gorham, St. John). - S a 1 i e $n$ t i a : Bufonidae (COOK, Ottawa).

Reptilia: S a u r i a : Iguanidae (ETHERIDGE, San Diego); Lacertidae (BÖHME, Bonn, \& WERMUTH, Ludwigsburg); Teiidae (HOTZ, Zürich, \& WERMUTH, Ludwigsburg); Amphisbaenidae (GANS, Ann Arbor, \& WERMUTH, Ludwigsburg); Scincidae (FUHN, Bukarest). S e r p e n t e s: Aniliidae, Uropeltidae, Xenopeltidae, Acrochordidae (RIEPPEL, Basel); Elapidae (KLEMMER, Frankfurt am Main, \& MARX, Chicago); Viperidae, Crotalidae (HAHN, Cottonwood/HOGE, São Paulo [?]). 